See discussions, stats, and author profiles for this publication at: https://www.researchgate.net/publication/327820643

\title{
Optimization of Neuropsychological Scores at the Baseline Visit Using Evolutionary Technique
}

Conference Paper · December 2017

DOI: 10.1109/WIECON-ECE.2017.8468891

CITATIONS

4

4 authors:

Vinutha. N

University Visvesvaraya College of Engineering

11 PUBliCATIONS 13 CITATIONS

SEE PROFILE

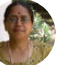

P. Deepa Shenoy

University Visvesvaraya College of Engineering

178 PUBLICATIONS 1,296 CITATIONS

SEE PROFILE

Some of the authors of this publication are also working on these related projects:

Project time synchronization View project

Project Efficient Morphometric Techniques in Alzheimer's Disease Detection: Survey and Tools View project
READS

26

Sonu Sharma

2 PUBLICATIONS 4 CITATIONS

SEE PROFILE

Venugopal K R

University Visvesvaraya College of Engineering

925 PUBLICATIONS 3,702 CITATIONS

SEE PROFILE 


\title{
Optimization of Neuropsychological Scores at the Baseline Visit Using Evolutionary Technique
}

\author{
Vinutha N, Sonu Sharma, \\ P Deepa Shenoy, Venugopal K R \\ Department of Computer Science and Engineering, \\ University Visvesvaraya College of Engineering, Bangalore University, \\ vinutha1v@gmail.com
}

\begin{abstract}
The neuropsychological battery of scores, are the measures of cognitive domains of human brain, that are considered as important features to distinguish healthy subjects from the subjects, suffering from Mild Cognitive Impairment (MCI). The instances of about 5542, with four time visits are separated from the total collected instances of the National Alzheimer's Coordinating Center (NACC) database. The analysis of the selected data shows that the large number of subjects is identified for 66-75 and 76-85 age groups. The Genetic Algorithms (GA) applied on the neuropsychological scores at the baseline visit, selects the best subset of scores required for the clinical diagnosis, and these scores are evaluated by the logistic regression model using Area Under Curve (AUC), accuracy and Mean Squared Error (MSE) as the metric. Simulations result show that a highest classification accuracy of $0.9427, \mathrm{AUC}$ of 0.9713 and less error rate of 0.041 is achieved for the combination of four neuropsychological scores Global Staging of Clinical Dementia Rating (CDRGLOB), Geriatric Depression Scale (GDS), Logical Memory Delayed (MEMUNITS), Digit Span Forward Length (DIGIFLEN). These scores are predominantly selected by the GA across many runs and thus have significant role for screening MCI subjects at the baseline visit.
\end{abstract}

Keywords-Cognitive Domains; Genetic Algorithm; Logistic Regression; Mild Cognitive Impairment; Neuropsychological Tests.

\section{INTRODUCTION}

Mild Cognitive Impairment refers to the early stage of dementia. The progression from MCI to Alzheimer's Disease (AD) or other types of dementia can be delayed by early detection and diagnose them with suitable disease modifying agents. Thus, the recognition of MCI is the most challenging research issue in the field of Neuropsychology.

To identify the early stage of dementia, there is tremendous increase in the development of advanced imaging technologies. These imaging technologies are essential for the structural and functional analysis of the brain. The significant changes associated with these images during the disease state can be further validated with the help of neuropsychological tests, those are also required to assess the mental status of a person during the clinical trials and drug development. Many studies conducted in the past, evaluated that the diagnosis is improved further through the combination of image biomarkers with neuropsychological tests, fluid markers and genetic markers. Thus, there is continuous effort for the development of various neuropsychological tests.
The impairment of the dementia subjects under various cognitive domains are measured by the standardized neuropsychological tests and further they are utilised to characterize the disease status of the affected person. The common domains affected in the $\mathrm{AD}$ subjects are episodic memory, semantic memory, working memory, executive function, visuospatial function, language, depression and dementia severity. There exists multiple neuropsychological tests to assess the mental status of a person in each specific domain. This results in the accumulation of large amount data and increases the cost of health-care system. Therefore machine learning algorithms $[1,2]$ are utilised to predict the significant features required for the clinical diagnosis.

\section{Contribution}

The instances with four time visits are separated from the NACC dataset. Further, we identified the age groups with maximum number of instances for the separated file. This is followed with the optimization of neuropsychological scores through GA approach and identification of cognitive domain impairments for the derived subset of scores.

\section{Motivation}

The cost of the healthcare systems has raised in recent times due to the development of multiple cognitive tests. These tests are the measure of cognitive domain in the $\mathrm{MCI} / \mathrm{AD}$ patients. The multiple cognitive tests has lead to an exponential growth of the clinical data. This results in the increase of computational complexity for computer aided diagnosis. Thus, identifying a small subset of scores, that contributes to early diagnosis are necessary for the clinical studies, and utilising these reduced feature set in the correlation studies, gives a high impact towards the disease prediction.

\section{Organisation}

The rest of the paper is organized as follows: Section II provides the literature survey, Sections III describes the methodology. Section IV presents the experiments and results. The paper concludes in Section V.

\section{LITERATURE SURVEY}

The survey discusses about the findings of the researchers on neuropsychological battery of scores. Barrens et al., [3] evaluated the tests for determining the correlation between age and $\mathrm{AD}$. The data for the test was collected from NACC, which was further divided based on the age groups. These subgroups were subjected to memory and non-memory tests. 
The outcomes derived from the linear regression analysis, shows that different neuropsychological scores are selected based on the increasing age group. Along with the identification of cognitive domain impairment, the behavioral symptoms are also observed at the initial stages of $\mathrm{AD}$, and these symptoms increases gradually for the older age group. From the results it is inferred that, the older aged people were more prone to psychosis (loss of contact with reality).

The authors in paper [4] performed a comparison between $\mathrm{MCI}$ in $\mathrm{AD}$ and Parkinson Disease (PD) by using the longitudinal data from NACC. The clinical and neuropsychological scores of participants having an Apolipo Protein E (APOE) allele greater than or equal to one are subjected to multi variable linear regression model for both $\mathrm{AD}-\mathrm{MCI}$ and PD-MCI patients. The results indicate that the conversion of MCI to dementia took longer time in PD when compared to AD. Hence it can be inferred that different tools should be used to diagnose dementia in $\mathrm{AD}$ and $\mathrm{PD}$.

In paper [5], the authors predicted $\mathrm{AD}, \mathrm{MCI}$ and normal subjects by applying threshold method on Logical Memory (LM) and Mini Mental State Examination (MMSE) tests. The clinical data for the tests was obtained from NACC database. The MMSE score is mapped on to various domains such as attention, orientation, naming, concentration, visuoconstruction and verbal learning whereas LM was used as a standardized measure for recalling capacity. The results showed that the accuracy obtained from the above tests are found to be limited. Hence there is no clear distinction between $\mathrm{MCI}$ and $\mathrm{AD}$ and also with normal cognition and MCI.

\section{Methodology}

The proposed methodology consists of four modules as shown in Fig. 1. It begins with the collection of data from NACC dataset. Then the data is pre-processed to select the instances with minimum of four visit times. For these selected instances, we considered the neuropsychological scores that measure cognitive domain in patients affected with AD. Further, these scores are subjected to GA to obtain subset of scores and evaluated through Logistic Regression model.

\section{A. Data Collection}

The data used in this study, comprised of the subjects from NACC. The development of NACC database is through the contribution of clinical data by several Alzheimer's disease Centers (ADC's) [6]. The study data has 11,734 unique instances and 467 features for both normal cognition and MCI subjects. The features collected for all the instances comprises of subject demographics, health history, neuropsychological battery summary scores, geriatric depression scale, global staging - clinical dementia rating, functional activities questionnaire, neuropsychiatric inventory questionnaire, clinician judgement of symptoms and clinical diagnosis.

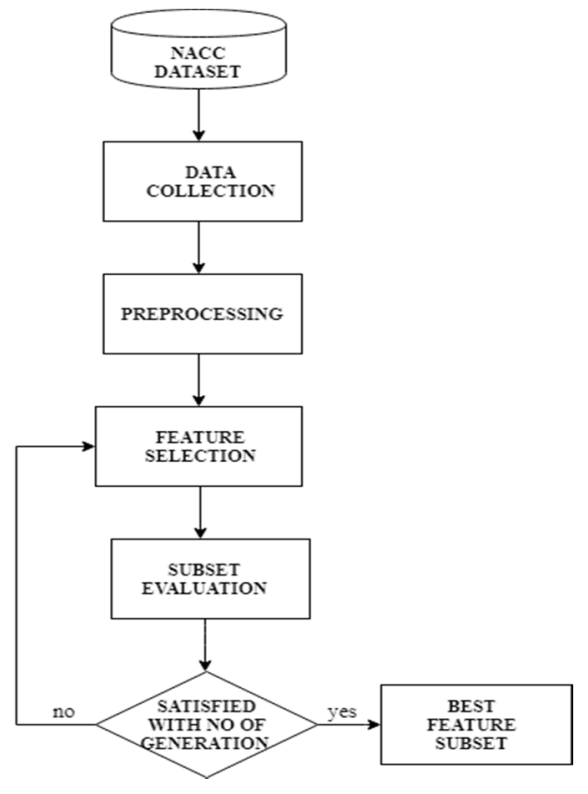

Fig. 1. Proposed Methodology

\section{B. Preprocessing}

The collected instances from NACC, has the number of visits varying from one to twelve. We filtered the dataset to contain only the patients who have visited for four times as shown in Fig. 2. The separated instances has the longitudinal data for minimum of 36 months to 48 months. This selection criteria is decided according to the requirement for the longitudinal studies.

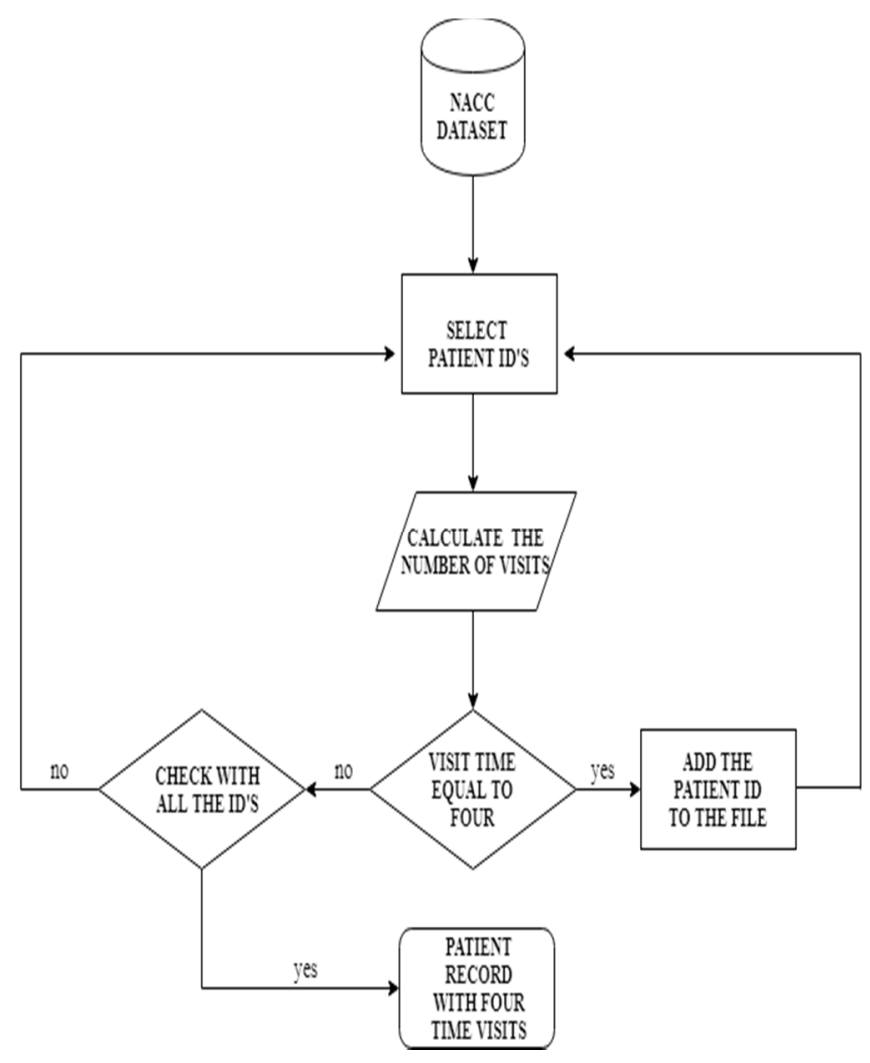

Fig. 2. Separation of the Instances with Four Visit Times 
In Fig. 2. , the unique ID of the patients is selected from the database. This is followed with the calculation of number of visits available for each patient. Then, we group and select the instances for four time visits. After the data separation, neuropsychological battery of scores is subjected to feature selection and evaluation.

The neuropsychological battery of scores measures the cognitive deficits in an individuals affected with AD. Thus, there is continuous effort in ADC's towards the development of new neuropsychological battery of scores. But these new scores has the score value of -4 , which indicates that UDS could not collect the information from different Alzheimer's Disease Center. Therefore, the scores considered for the study are : Clinical Dementia Rating Sum of Boxes (CDR SUM), Logical Memory Immediate (LOGIMEM), Digit Span Forward and Backward Test (DIGIF and DIGIB), Digit Span Forward Length and Backward Length (DIGIFLEN and DIGIBLEN), Wechsler Adult Intelligence Scale (WAIS), Trail Making Tests A and B (TRAIL A and TRAIL B), Category Fluency Tests (ANIMALS and VEG), CDR GLOBAL, MEMUNIT, GDS, MMSE and clinician diagnosis. Among these above mentioned scores, it is important to predict a small subset of scores with the highest AUC value, that is explained in next section.

\section{Feature Selection}

Genetic algorithms (GA) [7], [8] is a population based heuristic search technique. The optimal solution is obtained from GA as a result of three genetic operations such as selection, crossover and mutation.

\section{Algorithm I: Feature Selection by Genetic Algorithm}

Step1: Initialize the population

Step2: Select the population through tournament selection.

Step3: From the selected individual (in step2) generate new population by performing crossover and mutation operations.

Step4: Apply Elitism

Step5: Evaluate individuals from population using (1) for every generation using fitness function

Step6: Terminate GA at fixed number of generation

Each individual in the population has a feature set with sixteen cognitive tests, which are represented by a string of real numbers. Algorithm I begins with the population initialization followed by the selection technique. There exists different types of selection techniques such as rank based selection, roulette wheel selection and tournament selection. We choose tournament selection to search the first individual with the highest fitness value, among the randomly picked population based on the tournament size. Then, the selection of second best individual is also performed in a similar way. These two selected individuals from the current generation are subjected to crossover and mutation operations to generate a new improved population for the next generation. In the evolution of the new population, there are chances of missing the best individual from the previous generation. Therefore, the elitism in GA ensures that the fittest individual is selected in every generation as a substitute of least fit individual. The same process continues for many generation, until it meets some termination criteria. As GA is evolutionary based algorithm there should be some stopping criteria to be defined. The number of generation is chosen as stopping criteria in our study.

\section{Evaluation}

Logistic Regression is a prediction model, used to find the relation between the dependent (response) and independent (predictor) variable. The dependent variable considered in our study are Normal and MCI patients and neuropsychological scores as independent variables. The probability of predicting the response variable through the logistic regression is based on the number of independent variables selected by GA and regression coefficients as defined in equation (1).

$$
\mathrm{Q}=\frac{e^{\beta 0+\mathrm{P} 1 \beta 1+\mathrm{P} 2 \beta 2, \ldots . \mathrm{Pn} \beta \mathrm{n}}}{1+e^{\beta 0+\mathrm{P} 1 \beta 1+\mathrm{P} 2 \beta 2, \ldots . \mathrm{Pn} \beta \mathrm{n}}}
$$

Where $\mathrm{Q}$ is the response variable, $\mathrm{P}=\left[\mathrm{P}_{1}, \mathrm{P}_{2} \ldots \mathrm{P}_{\mathrm{n}}\right]$ is a set of predictor variables and $\beta=\left[\beta_{0}, \beta_{1}, \beta_{2, \ldots} \beta_{\mathrm{m}}\right]$ is a set of regression co-efficient. The output obtained from the logistic regression is assessed using AUC as the fitness function. Higher the AUC, the better is the predictive performance of the model.

\section{EXPERIMENTS AND RESULTS}

The data analysis for the four times visited subjects are shown in Fig. 1.

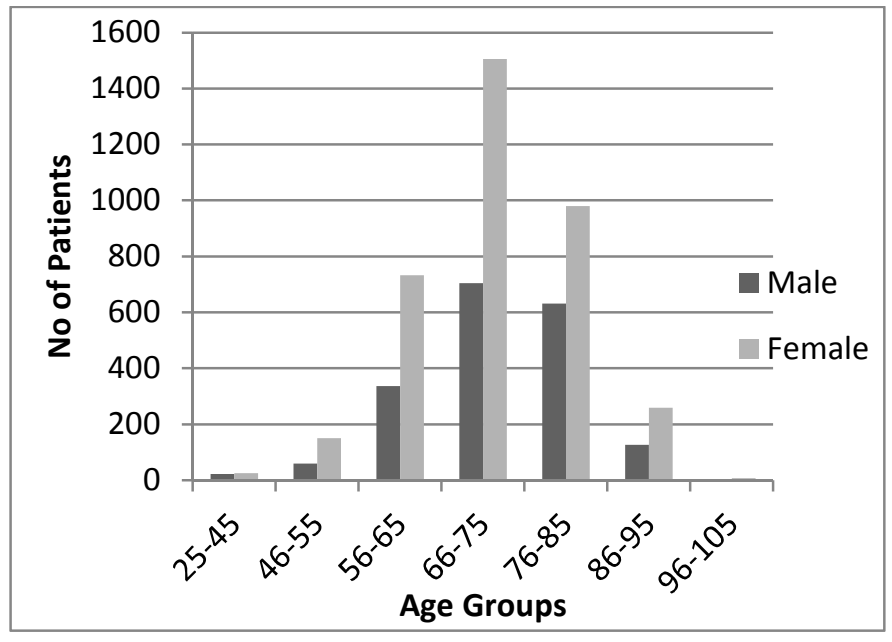

Fig.3. Data Analysis for the Instances with Four Time Visits

From the Fig. 3. it is inferred that the NACC data comprises of the individuals for different age groups. The range of age groups varies from 25 to 105 years old. Through the comparison of different age groups, it is observed that the 
number of subjects are maximum in $66-75$ and $76-85$ age groups and also comprises more number of female subjects.

The parameters considered for simulations in GA are:

Population: 50

Mutation Rate: 0.1

Crossover Rate: 0.9

Elitism: 2

Number of generation: 10

In GA, the crossover rate with high probability of 0.9 indicates the best individuals are combined to produce better offspring in the next generation. Whereas, the mutation rate with lower probability of 0.1 allows the new individual to have small changes within the chromosome. By following these two steps, the new improved population is obtained for every generation and these are evaluated through the prediction models based on the fitness function. The elitism with 2 ensures that the two best individuals are selected from previous generation to substitute the least fit individuals from present generation. Finally, GA is terminated with no of generation equal to 10 because of consistent results.

With the consideration of above mentioned parameters, search for the subset of features is performed by GA on the baseline data having MCI and Normal subjects. The selected features with the ratio of 60:40 as training and test samples are subjected to the prediction model. With this ratio, the simulations are carried out for multiple times to identify the best combination of small subset of scores with the highest predictive performance. The performances are evaluated by AUC, MSE and accuracy.

TABLE I. Performance Evaluation of Feature Subsets Obtained by Genetic Algorithm

\begin{tabular}{|c|c|c|c|c|}
\hline $\begin{array}{l}\text { Feature } \\
\text { Number }\end{array}$ & $\begin{array}{c}\text { Neuropsychological } \\
\text { Scores }\end{array}$ & AUC & Accuracy & MSE \\
\hline 1 & CDRSUM & 0.947 & 0.9265 & 0.057 \\
\hline 2,6 & $\begin{array}{l}\text { CDRGLOB, } \\
\text { MEMUNITS }\end{array}$ & 0.967 & 0.9403 & 0.042 \\
\hline $2,4,6$ & $\begin{array}{l}\text { CDRGLOB, NACC } \\
\text { MMSE, MEMUNITS }\end{array}$ & 0.968 & 0.9393 & 0.043 \\
\hline $2,3,6,8$ & $\begin{array}{l}\text { CDRGLOB, NACC } \\
\text { GDS, MEMUNITS, } \\
\text { DIGIFLEN }\end{array}$ & 0.971 & 0.9427 & 0.041 \\
\hline $\begin{array}{c}8,3,2,6 \\
10\end{array}$ & $\begin{array}{l}\text { DIGIFLEN, NACC } \\
\text { GDS, CDR GLOB, } \\
\text { MEMUNITS, } \\
\text { DIGIBLEN }\end{array}$ & 0.971 & 0.9427 & 0.042 \\
\hline
\end{tabular}

The features represents the cognitive test scores of commonly affected domains in dementia subjects. The results from the simulations show that the better performance is obtained for all 16 cognitive tests with AUC of 0.969. But our aim is to identify a subset of scores with the highest predictive performance. Thus, we perform simulations and evaluate the performance for a single feature to maximum of 5 features.

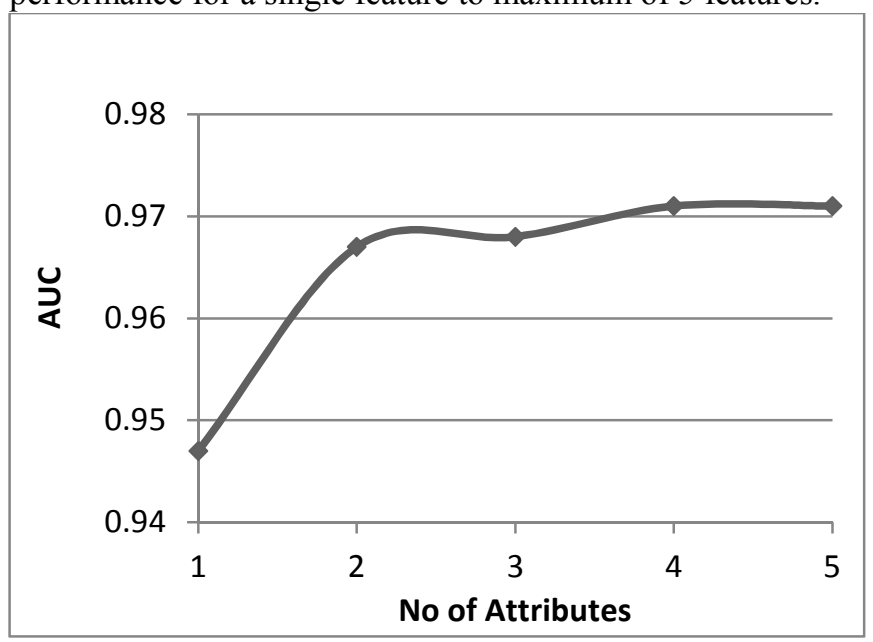

Fig. 4. Comparison of Performance Analysis based on Number of Attributes

The results shown in Fig. 4. Indicates that the feature number 1 which is a measure of dementia severity results with the AUC of 0.947 , therefore it is a good predictor when used as a single variable. When a set of two variables were considered, the result was improved with AUC of 0.967 for the feature number 2 and 6 . These variables are a measure of long term memory. The simulations is continued further with the increasing number of features and it is observed that the tests related to short term memory and depression are also predicted along with long term memory for the combination of three, four and five subset of features. There are some of the features which are repeatedly selected by GA across different runs. As shown in Table I. the most common features predicted by the model are 2, 3, 6, 8 and the combination of these four set of features results with AUC of 0.971 and highest accuracy of 0.942 and MSE of 0.041 . Therefore, it is inferred that, the memory domain are impaired for the MCI patients at the baseline visit.

\section{CONCLUSIONS AND FUTURE WORK}

The 16 cognitive tests scores for four times visited patients are collected from the NACC database. These test scores subjected to the GA, reduces its number and identify the subset of scores with highest predictive power. The simulations performed on the baseline data show that the highest performance is obtained for the combination of four set of scores. These scores are a measure of short term and long term memory and depression. Therefore, the predicted results indicate, the subjects at the baseline visit are commonly affected at their memory domain. In future, we plan to perform prediction for the follow-up visits and 
perform comparison of the predicted scores between the baseline and follow-up visits, and also include scores related to behavioral and functional domain.

\section{ACKNOWLEDGMENT}

The authors would like to thank curators of the NACC database for providing the data to conduct the research.

\section{REFERENCES}

[1] Sandhya Joshi, P Deepa Shenoy, Venugopal K R, L M Patnaik, "Classification of Neuro Degenerative Disorder based on Major Risk Factors emphasing Machine Learning Techniques", International Journal of Computer Theory and Engineering(IJCTE), ISSN : 17938201, vol. 2, no. 4, pp. 350-356, 2010.

[2] Sandhya Joshi, P Deepa Shenoy, Venugopal K R, L M Patnaik, "Classification of Treatment of Different Stages of Alzheimer's Disease using Machine Learning Methods", International Journal of Bioinformatics Research, ISSN : 0975-3087, vol. 2, no. 1, pp. 44-52, 2010.

[3] J. Barnes, B. C. Dickerson, C. Frost, L. C. Jiskoot, D. Wolk, and W. M. van der Flier, "Alzheimer's Disease First Symptoms are Age Dependent: Evidence from the NACC Dataset," Alzheimer's Dementia, vol. 11, no. 11, pp. 1349-1357, 2015.

[4] L. M. Besser, I. Litvan, S. E. Monsell, C. Mock, S. Weintraub, X.-H. Zhou, and W. Kukull, "Mild Cognitive Impairment in Parkinson's Disease versus Alzheimer's Disease," Parkinsonism Related Disorders, vol. 27, pp. 54-60, 2016.

[5] K. R. Chapman, H. Bing-Canar, M. L. Alosco, E. G. Steinberg, B. Martin, C. Chaisson, N. Kowall, Y. Tripodis, and R. A. Stern, "Mini Mental State Examination and Logical Memory Scores for Entry into Alzheimer's Disease Trials," Alzheimer's Research Therapy, vol. 8, no. 1, p. 9, 2016.

[6] K. Steenland, J. Macneil, S. Bartell, and J. Lah, "Analyses of Diagnostic Patterns at 30 Alzheimer's Disease Centers in the US," Neuroepidemiology, vol. 35, no. 1, pp. 19-27, 2010.

[7] L. Vandewater, V. Brusic, W. Wilson, L. Macaulay, and P. Zhang, "An Adaptive Genetic Algorithm for Selection of Blood-Based Biomarkers for Prediction of Alzheimer's Disease Progression," BMC Bioinformatics, vol. 16, no. 18, 2015.

[8] Srinivasa K G, Venugopal K R and L M Patnaik, "An Efficient Stock Market Prediction usingFuzzy based Neuro-Genetic Algorithm", International Journal of Hybrid Intelligent Systems, IOS Press, e-ISSN : 1875-8819, p-ISSN : 1448-5869, vol. 3, no 2, pp. 63-81, July 2006. 